\title{
Differences in trends in estimated incidence of myocardial infarction in non-diabetic and diabetic people: Monitoring Trends and Determinants on Cardiovascular Diseases (MONICA)/Cooperative Health Research in the Region of Augsburg (KORA) registry
}

\author{
A. Icks • T. Dickhaus • A. Hörmann • M. Heier • \\ G. Giani • B. Kuch • C. Meisinger
}

Received: 9 February 2009 / Accepted: 4 June 2009 /Published online: 15 July 2009

(C) Springer-Verlag 2009

\begin{abstract}
Aims/hypothesis One major objective of the St Vincent Declaration was to reduce the excess risk of myocardial infarction in patients with diabetes mellitus. We estimated the trend of the incidence and relative risk of myocardial infarction in the diabetic and non-diabetic populations in southern Germany from 1985 to 2006.
\end{abstract}

A. Icks $(\bowtie) \cdot$ T. Dickhaus $\cdot$ G. Giani

German Diabetes Center, Institute of Biometrics and

Epidemiology, Heinrich-Heine-University,

Leibniz-Centre for Diabetes Research,

Auf'm Hennekamp 65,

40225 Düsseldorf, Germany

e-mail: icks@ddz.uni-duesseldorf.de

\footnotetext{
A. Hörmann

Helmholtz Zentrum München, German Research Center

Neuherberg, Germany

M. Heier $\cdot$ C. Meisinger

Central Hospital of Augsburg,

MONICA/KORA Myocardial Infarction Registry,

Augsburg, Germany

M. Heier $\cdot$ C. Meisinger

Helmholtz Zentrum München, German Research Center

Neuherberg, Germany

B. Kuch

Department of Internal Medicine 1, Cardiology,

Central Hospital of Augsburg,

Augsburg, Germany
}

for Environmental Health $(\mathrm{GmbH})$, Institute of Health Economics,

for Environmental Health $(\mathrm{GmbH})$, Institute of Epidemiology,
Methods Using data from the Monitoring Trends and Determinants on Cardiovascular Diseases (MONICA)/ Cooperative Health Research in the Region of Augsburg (KORA) Project in southern Germany, we ascertained all fatal and non-fatal first myocardial infarctions between 1985 and 2006 ( $n=14,891$, age 25-74 years). We estimated the diabetic and the non-diabetic populations using data on diabetes prevalence from surveys, and evaluated incidence of myocardial infarction in the two estimated populations. To test for time trends, we fitted Poisson regression models.

Results Of individuals with first myocardial infarction, $71 \%$ were male and $28 \%$ known to have diabetes. In the non-diabetic population, myocardial infarction incidence decreased by about $1.5 \%$ to $2.0 \%$ per year. A comparable decrease was seen in the population of diabetic women. However, in the population of diabetic men, incidence of myocardial infarction increased by about $1 \%$ per year. Over the whole study period, myocardial infarction incidence decreased by $34 \%$ and $27 \%$ in non-diabetic men and women respectively (RR $0.66,95 \%$ CI $0.59-0.74$ and 0.73 , $0.62-0.87$ respectively). In diabetic women, it decreased by $27 \%$ (RR 0.73, 0.61-0.88), whereas in diabetic men, it increased by $25 \%$ (RR 1.25, 1.07-1.45).

Conclusions/interpretation Our results suggest that the $\mathrm{St}$ Vincent goal of reducing excess cardiovascular morbidity in diabetic individuals has not been achieved and that the situation in men has actually got worse.

Keywords Diabetic and non-diabetic population . Myocardial infarction - MONICA registry · Trend 


$\begin{array}{ll}\text { Abbreviations } \\ \text { KORA } & \begin{array}{l}\text { Cooperative Health Research in the Region of } \\ \text { Augsburg }\end{array} \\ \text { MI } & \begin{array}{l}\text { Myocardial infarction } \\ \text { MONICA }\end{array} \\ & \begin{array}{l}\text { Monitoring Trends and Determinants on } \\ \text { Cardiovascular Diseases }\end{array}\end{array}$

\section{Introduction}

In 1989, the St Vincent Declaration defined a number of major targets for the improvement of diabetes care [1]. As several studies have confirmed an increased risk of CHD and cardiovascular disease events in people with diabetes [2, 3], narrowing of the gap in cardiovascular morbidity between the diabetic and non-diabetic populations was one of the primary objectives [1]. Several studies from western Europe and the USA indicate that incidence of CHD and cardiovascular events, e.g. MI, is declining [4]. However, it is unknown whether the decline in cardiovascular events has also been observed in people with diabetes or whether the gap between the diabetic and non-diabetic populations has narrowed.

Several studies have examined the secular trends in cardiovascular mortality in people with and without diabetes [5-10], but few have been able to compare cardiovascular event rates. Analyses from Norway and the US have been based on cross-sectional surveys or hospital discharge data [11, 12]. Only one study to date has evaluated the trend of MI incidence in diabetic and nondiabetic individuals, finding favourable trends in the latter, but not in diabetic individuals [13].

In Germany from 1985 to 1994, the incidence of MI in 25to 74-year-old diabetic individuals was found to have increased 3.7-fold in men and 5.9-fold in women, compared with non-diabetic individuals in the same age range [14]. Several efforts have been undertaken in Germany to improve diabetes treatment and thus reduce cardiovascular morbidity in diabetic individuals, but it is unknown whether MI risk in diabetic compared with non-diabetic individuals has changed. Thus, the aim of our study was to estimate time trends for the incidence of MI in diabetic and non-diabetic individuals in a region of southern Germany using a population-based registry. The study is part of an ongoing initiative in Germany, aiming to evaluate all six St Vincent objectives, i.e. (in addition to $\mathrm{MI}$ incidence) reduction of amputation, blindness, end-stage renal disease, stroke and worse pregnancy outcomes.

\section{Methods}

Study population and data assessment We used data from Monitoring Trends and Determinants on Cardiovascular diseases (MONICA)/Cooperative Health Research in the Region of Augsburg (KORA) population surveys and from the MONICA/KORA myocardial infarction registry in Augsburg, Germany, to define the diabetic and nondiabetic population background and to identify cases with fatal or non-fatal MI. Augsburg and its surrounding areas have a total population of about 600,000 . The population-based Augsburg Coronary Event Registry was implemented in October 1984 as part of the WHO MONICA project [15-18]. Since 1996 the registry has been run within the framework of KORA. All cases of CHD deaths and non-fatal MI in the 25- to 74-year-old study population in Augsburg and the Augsburg and Aichach-Friedberg regions (about 400,000 inhabitants) are continuously registered [19]. Data sources for hospitalised patients include eight hospitals within the study region and two hospitals in adjacent areas. Methods of casefinding, diagnostic classification of events and data quality control have been described elsewhere $[15,17]$. Up to 31 December 2000 non-fatal MI was registered on the basis of diagnostic criteria in the WHO-based MONICA protocol [15]. Since 1 January 2001 all patients with MI diagnosed according to European Society for Cardiology and American College of Cardiology criteria, including troponin measurement, have also been registered [20]. Briefly, patients surviving for at least $24 \mathrm{~h}$ (non-fatal MI) were interviewed while in hospital using a standardised questionnaire and further data were gathered in a concluding chart review. CHD deaths were identified through the regional health offices by checking all death certificate diagnoses giving suspected CHD as main cause of death. In addition, a written questionnaire was routinely sent to the last physician to treat the deceased person and to the coroner to establish disease history, risk factors, prior medication and circumstances of death (mean response $85 \%$ ). MONICA diagnostic criteria define early CHD death as CHD deaths occurring before admission to hospital or within $24 \mathrm{~h}$ of hospitalisation. For this analysis, we only used the first infarction per patient.

In addition we also used data from four population-based surveys conducted in the Augsburg region between 1984 and 2001 (1984/85, 1989/90, 1994/95 and 1999/2001). The survey population was in the 25 to 74 years age range and was surveyed using cluster sampling procedures [19]. The response rate was between 68 and $79 \%$. Diabetes was assessed by self-report of a physician's diagnosis of diabetes or by use of glucose-lowering medication. The diabetes prevalence was $3.7 \%$ to $4.6 \%$ in men and $3.5 \%$ to $3.7 \%$ in women. No significant time trend was found [21], corresponding to findings for the whole of Germany [22].

Statistical analysis The main analyses were performed separately for each sex. 
We stratified the study population in three layers according to age (25-54, 55-64 and 65-74 years of age), sex and calendar year (1985-2006). The population with diabetes in each resulting stratum was estimated by obtaining the population of each stratum within the study area from the regional statistic office and multiplying this by the age- and sex-specific prevalence of diabetes, obtained from the four MONICA/KORA surveys that were carried out in 1984, 1989, 1994 and 1999/2000. As no significant time trend in diabetes prevalence was evident [21], the averaged estimate for diabetes prevalence over the four surveys was used. For men in the age groups 25 to 54 , 55 to 64 and 65 to 74 years, diabetes prevalence was: $1.6 \%$ (95\% CI 0.6-2.6), 7.9\% (6.2-9.6) and 10.7\% (10.1-11.4), respectively; for women (same age groups) it was: $1.2 \%$ (0.4-2.0), 6.7\% (6.0-7.3) and 10.2\% (6.6-13.7).

Stratum-specific and directly standardised incidence rates of MI (standard population: West German population 1987) were estimated for each calendar year for the total, estimated diabetic and estimated non-diabetic populations, respectively.

To test for time trends, we first fitted separate (sexspecific) Poisson regression models with incidence of MI as dependent variable for diabetic and non-diabetic individuals, using year of MI diagnosis registration (linear continuous difference from baseline year 1985) and age (three strata) as independent variables. Next, and in addition, we fitted similar common Poisson regression models to the entire population. In these models, an additional variable was included for diabetes, as well as an interaction term for diabetes and years since 1985. Finally, we evaluated differences in incidence trends between younger and older individuals (25-64 vs 65-74 years of age) by including interaction terms for age group with year difference from baseline. These analyses were again performed separately for each sex and for diabetes and non-diabetes status.

To take into account overdistribution of the dependent variable, we performed all analyses using de-scale adjustment [23]. All analyses were performed using Statistical Analysis Systems (SAS for Windows 2000, Release 9.1; SAS Institute, Cary, NC, USA).

\section{Results}

Between 1985 and 2006, 14,891 individuals with a first MI were registered. We excluded 313 individuals as their diabetes status was not known. Of the remaining 14,578 individuals with MI, $71 \%$ were men and $28 \%$ were known to have diabetes mellitus. Characteristics of individuals with MI are shown in Table 1. The majority of MIs occurred in the elderly. Mean ages at diagnosis were higher
Table 1 Description of the MI population, Augsburg, MONICA region, 1985-2006

\begin{tabular}{llllll}
\hline Variables & \multicolumn{2}{l}{ Men } & & \multicolumn{2}{l}{ Women } \\
\cline { 2 - 3 } \cline { 5 - 6 } & Diabetes & No diabetes & & Diabetes & No diabetes \\
\hline$n$ & 2,635 & 7,694 & & 1,497 & 2,752 \\
Mean age, & $63.3(8.2)$ & $59.9(9.9)$ & & $66.8(6.7)$ & $64.2(8.8)$ \\
years (SD) & & & & \\
Age groups (\%) & & & & 14.9 \\
25-54 years & 15.3 & 28.4 & 6.3 & 26.1 \\
$55-64$ years & 32.7 & 33.2 & & 21.7 & 59.0 \\
$65-74$ years & 52.0 & 38.4 & & 71.9 & 59.9 \\
\hline
\end{tabular}

in women than in men and in diabetic than in non-diabetic patients.

Incidence of myocardial infarction and relative risks Figure 1 shows the standardised incidence rates for each year. There were brief oscillations, in particular in men. Over the whole study period, MI incidence declined in non-diabetic men and women, as well as in diabetic women; in diabetic men, MI incidence increased. In women, the RR of MI in the diabetic compared with the non-diabetic population (expressed as comparative incidence figures from direct standardisation) ranged from 5.11 (95\% CI 3.41-7.67) in 2003 to $11.46(7.82-16.81)$ in 2001, with no significant trend during the study period. In men, RR was between 3.71 (2.71-5.09) in 1987 and 8.86 (6.90-11.37) in 2000, increasing significantly during the observation period $(p<0.001)$.

Trend results from the Poisson regression are given in Table 2. Models 1a and $1 \mathrm{~b}$ present RRs in the diabetic and the non-diabetic populations. In the latter, we found an estimated decrease of MI of about $1.5 \%$ to $2.0 \%$ per year. In diabetic women, there was a comparable decrease. In diabetic men, by contrast, the incidence of $\mathrm{MI}$ increased by about $1 \%$ per year. The interaction between diabetes and calendar year trend was significant in men, as can be seen in Model $2(p<0.001)$ (Table 2).

The increase of MI in diabetic men was particularly marked in the age groups up to 64 years, but not in 65 to 74-year-old individuals, with RRs per age group as follows: 25-54 years: RR 1.02, 95\% CI 1.01-1.04; 55-64 years: RR $1.02,1.01-1.03$; 65-74 years: RR 1.00, 0.99-1.01; however, the interaction between incidence trend and age was not quite significant $(p=0.06)$.

Over the whole study period, the incidence of MI decreased by $34 \%$ and $27 \%$ in non-diabetic men and women (RR 0.66, 0.59-0.74 and 0.73, 0.62-0.87), respectively. In diabetic women, it decreased by $27 \%$ (RR 0.73 , $0.61-0.88$ ), whereas in diabetic men it increased by $25 \%$ (RR 1.25, 1.07-1.45). 


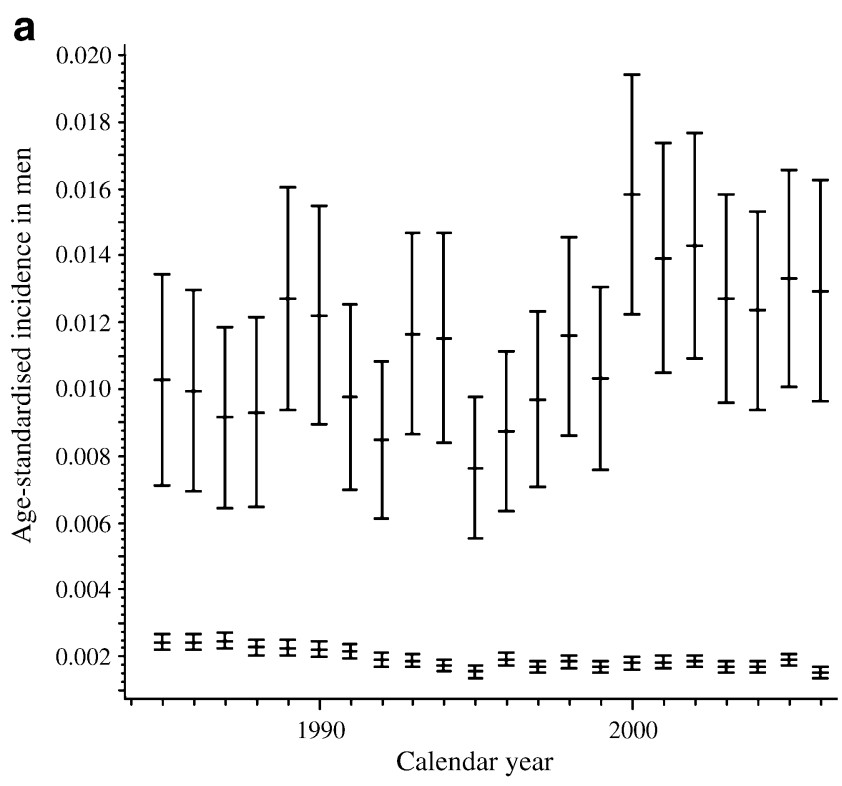

b

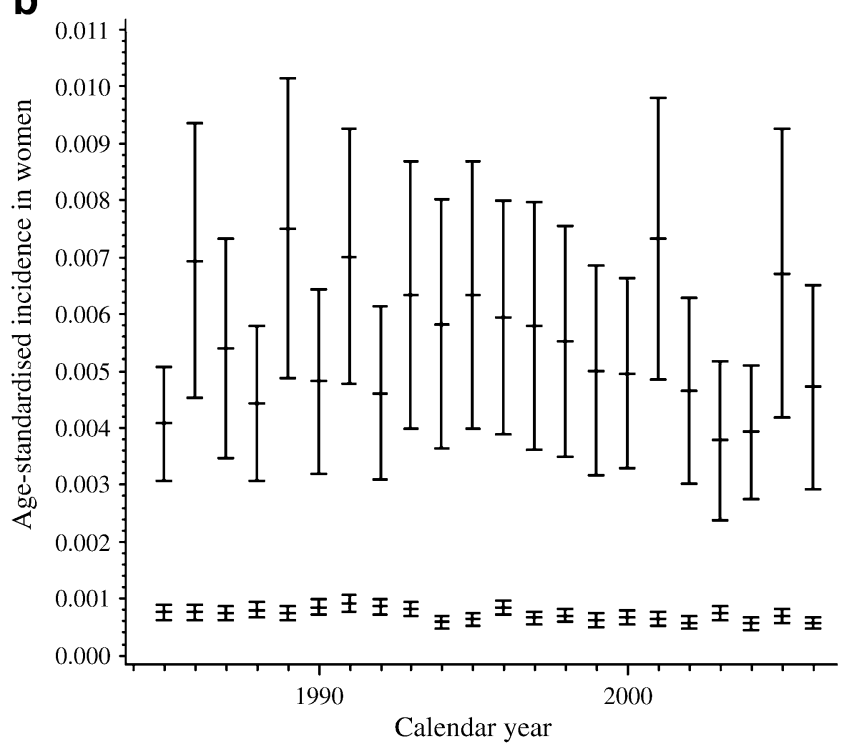

Fig. 1 Age-standardised incidence of MI in diabetic (top rows) and non-diabetic (bottom rows) participants, MONICA/KORA Augsburg 1985-2006, in (a) men and (b) women. Values were standardised to the German standard population

\section{Discussion}

Study findings and implications Our study is part of an evaluation of how well the St Vincent objectives have been met in Germany. In southern Germany, between 1985 and 2006, we found a significant decrease in the incidence of MI in non-diabetic men and women. In diabetic women, the incidence of MI also decreased significantly, whereas in diabetic men it increased significantly. Correspondingly, the $\mathrm{RR}$ for $\mathrm{MI}$ in the diabetic compared with the non-diabetic population remained stable in women, but increased in men. Thus, despite national programmes designed to improve diabetes care, e.g. disease management programmes, the reduction of excess cardiovascular morbidity in diabetic individuals as a declared main objective in the St Vincent declaration [1] has not been achieved. This applies in particular for diabetic men, in whom RR for MI was actually higher, especially in the younger and middle-aged age groups.

Speculating on the possible reasons, it is possible that trends in the prevalence of MI risk factors, e.g. obesity, or trends in healthcare for cardiovascular diseases were different for diabetic vs non-diabetic men. However, we were unable to find any such explanations in the Augsburg populations. Overall, the prevalence of obesity increased, whereas hypertension and dyslipidaemia tended to decrease [24]. The use of glucose-lowering, as well as antihypertensive and lipid-lowering agents increased in diabetic men and women [25]. Moreover, other changes in diagnostic or therapeutic strategies that possibly affect men and women differently may also have occurred. As diabetes mellitus is more frequent in women than men, the interaction between diabetes mellitus and CHD in men may have received less attention during the study period, but our analysis found no evidence for this in the study region.

Comparison with other studies Whereas a number of studies have evaluated the trend of cardiovascular mortality rates in diabetic and non-diabetic individuals [7, 9, 10], only a few have looked at event rates. In Norway, the percentage of diabetic and non-diabetic individuals with a history of MI did not change between 1984 and 1997; however, the results were based on self-reports from two cross-sectional surveys [12]. In the USA, a sharp increase in the number of diabetic patients hospitalised for MI was observed between 1988 and 2002, but the study was based on hospital discharge data and MI incidence in the diabetic and non-diabetic populations was not analysed [11]. Only one study has analysed the trend of MI incidence [13]. In contrast to our study, it found that the incidence of first MI decreased only in non-diabetic men, with a decline in the incidence of first MI seen neither in non-diabetic women, nor in diabetic men or women. Thus this Swedish study also showed a worsening in diabetic men as compared with diabetic women. Compared with Germany, the RRs for MI in diabetic compared with non-diabetic individuals were much higher in Sweden (RR approx. 8 and 15 for men and women, respectively) [13]. The authors of this study also failed to find an explanation for their findings. They speculate that diabetes as such may have a strong influence and is an independent risk factor for cardiovascular diseases, and that a residual risk remains despite improvements of other risk factors due to preventive interventions. However, this would not explain the differences between men and women. Interestingly, another Swedish study, which evaluated the trend of excess mortality rates ascribed 
Table 2 Results of Poisson models: relative risks for MI, Augsburg MONICA region, 1984-2006

\begin{tabular}{|c|c|c|}
\hline \multirow[t]{2}{*}{ Variables } & \multicolumn{2}{|c|}{ Relative risk for MI $(95 \% \mathrm{CI})$} \\
\hline & Men & Women \\
\hline \multicolumn{3}{|l|}{ Model 1a (diabetic) } \\
\hline Calendar year & $1.01(1.00-1.02)^{*}$ & $0.99(0.98-0.99)^{*}$ \\
\hline \multicolumn{3}{|l|}{ Age (years) ${ }^{\mathrm{a}}$} \\
\hline $55-64$ & $1.73(1.52-1.98)^{*}$ & $2.17(1.71-2.79)^{*}$ \\
\hline $65-74$ & $3.05(2.70-3.47)^{*}$ & $5.48(4.41-6.90)^{*}$ \\
\hline \multicolumn{3}{|l|}{ Model 1b (non-diabetic) } \\
\hline Calendar year & $0.98(0.98-0.99)^{*}$ & $0.99(0.98-0.99)^{*}$ \\
\hline \multicolumn{3}{|l|}{ Age (years) ${ }^{\mathrm{a}}$} \\
\hline $55-64$ & $4.97(4.58-5.40)^{*}$ & $6.61(5.64-7.76)^{*}$ \\
\hline $65-74$ & $9.07(8.37-9.83)^{*}$ & $18.00(15.65-20.79)^{*}$ \\
\hline \multicolumn{3}{|l|}{ Model 2} \\
\hline Calendar year & $0.98(0.97-0.99)^{*}$ & $0.99(0.98-0.99)^{*}$ \\
\hline Diabetes mellitus & $3.30(2.74-3.95)^{*}$ & $6.38(5.36-7.58)^{*}$ \\
\hline \multicolumn{3}{|l|}{ Age (years) ${ }^{\mathrm{a}}$} \\
\hline $55-64$ & $4.36(3.94-4.82)^{*}$ & $5.76(4.95-6.72)^{*}$ \\
\hline $65-74$ & $7.80(7.07-8.61)^{*}$ & $15.19(13.24-17.49)^{*}$ \\
\hline Diabetes $\times$ calendar year & $1.03(1.01-1.04)^{*}$ & $1.00(0.99-1.01)$ \\
\hline
\end{tabular}

${ }^{\text {a }}$ Baseline: $25-54$ years

$* p<0.05$

to diabetes, found the trend to be more advantageous in women than in men. The authors suggest that this might be explained by the greater willingness of women to follow their doctors' advice or by the fact that diabetic women smoke less than male counterparts [26].

Study limitations A number of limitations have to be considered. First, the results of our study are dependent on the estimates of the number of diabetic individuals in the background population and are based on the assumption that diabetes prevalence remained stable over time. There is evidence that this assumption is valid, since no significant change in diabetes prevalence was found in repeat surveys [21] or in other German surveys [22]. However, participation in these surveys decreased with diabetic individuals more likely to be non-participants [27]. Furthermore, a number of individuals are likely to have undiagnosed diabetes [27]. It is possible that the prevalence of undiagnosed diabetes decreased during the study period, due to higher disease awareness and diabetes screening. However, we have no data to suggest that these phenomena, if present, would explain the different trends in men and women. Nevertheless, we cannot rule out the possibility of bias.
Second, we did not include clinical variables, because in individuals with fatal MI (39\% of all MIs), these data are based on the patients' medical history and documentation of their routine clinical care.

Third, the definition of MI changed in 2000, since troponin measurement was included in the criteria list. This might have led to an inclusion of 'milder' MI cases [28] and such cases might be unevenly distributed in diabetic and non-diabetic individuals, or in men and women. However, when for 2001 to 2004 only MI cases with creatinine kinase of $>300 \mathrm{U} / 1$ (an indicator for MI severity) were included, the diabetes prevalence was not significantly lower (27\% vs 29\%), with no relevant difference between men and women (data not shown). In patients who died before reaching the hospital, the new definition is not applicable. Thus, there is no evidence that the changed definition could have affected diabetic or non-diabetic individuals or men and women in a substantially different way; the RRs therefore should be unbiased.

Fourth, in diabetic and non-diabetic men, the time trend was not linear (significant test on non-linearity, data not shown). Over short time periods, the increases and decreases were within the statistical uncertainties. We consider these non-linearities to be due to brief oscillations and not to structural deviations from an increase (nondiabetic men) or decrease (diabetic men) of the incidence course over time.

Fifth and last, we limited our analysis to individuals between 25 and 74 years of age, although many patients with $\mathrm{MI}$ and a large proportion of patients with diabetes are above this upper limit. However, the population-based surveys used as data source were limited to this age group and we preferred to use the region-specific estimates of diabetes prevalence from these well established KORA surveys.

Conclusions With regard to the objectives of the St Vincent Declaration, we found a decrease in incidence of first MI in the estimated populations of non-diabetic men, non-diabetic women and diabetic women. However, in the estimated population of diabetic men, especially in younger age groups, incidence was increased. Thus, our results suggest that the St Vincent goal of reducing excess cardiovascular morbidity in diabetic individuals has not been achieved and that the situation in men has actually become worse.

Acknowledgements This project was supported by a grant of the German Ministry of Health and the German Diabetes Foundation. The German Diabetes Centre is financed by the German Federal Ministry of Health and by the Northrhine-Westphalian Ministry of Science. The KORA research platform and the MONICA Augsburg studies were initiated and financed by the Helmholtz Zentrum München, German Research Centre for Environmental Health, which is funded by the German Federal Ministry of Education, Science, Research and Technology, and by the State of Bavaria. Since the year 2000 
myocardial infarction data collection has been co-financed by the German Federal Ministry of Health and Social Security to provide population-based myocardial infarction morbidity data for the official German Health Report (see www.gbe-bund.de, accessed 3 November 2008). We thank all members of the Helmholtz Zentrum München, Institute of Epidemiology and the field staff in Augsburg who were involved in the planning and conduct of the study. We wish to thank the local health departments and the private physicians of the study area, as well as the clinicians of the hospitals involved, for their support. Finally, we express our appreciation to all study participants.

Duality of interest The authors declare that there is no duality of interest associated with this manuscript.

\section{References}

1. Diabetes care and research in Europe (1989) The Saint Vincent Declaration. World Health Organization, ICP/CLR 034

2. Becker A, Bos G, de Vegt F et al (2003) Cardiovascular events in type 2 diabetes: comparison with non-diabetic individuals without and with prior cardiovascular disease. 10-year follow-up of the Hoorn Study. Eur Heart J 24:1406-1413

3. Tunstall-Pedoe $\mathrm{H}$, Kuulasmaa $\mathrm{K}$, Mähonen $\mathrm{M}$, Tolonen $\mathrm{H}$, Ruokokoski E, Amouyal P (1999) Contribution of trends in survival and coronary event rates to changes in coronary heart disease mortality: 10-year results from the 37 WHO MONICA Project populations. Lancet 353:1547-1557

4. Kuulasmaa K, Tunstall-Pedoe H, Dobson A et al (2000) Estimation of contribution of changes in classic risk factors to trends in coronary-event-rates across the WHO MONICA Project populations. Lancet 355:675-687

5. Chun BY, Dobson AJ, Heller RF (1997) The impact of diabetes on survival among patients with first myocardial infarction. Diabetes Care 20:704-708

6. Dale AC, Vatten LJ, Nilsen TI, Midthjell K, Wiseth R (2008) Secular decline in mortality from coronary heart disease in adults with diabetes mellitus: cohort study. BMJ 337:A236 Abstract

7. Gu K, Cowie CC, Harris MI (1999) Diabetes and decline in heart disease mortality in US adults. JAMA 281:1291-1297

8. Mukamal KJ, Nesto RW, Cohen MC et al (2001) Impact of diabetes on long-term survival after acute myocardial infarction: comparability of risk with prior myocardial infarction. Diabetes Care 24:1422-1427

9. Sprafka JM, Burke GL, Folsom AR, McGovern PG (1991) Hahn LP (1991) Trends in prevalence of diabetes mellitus in patients with myocardial infarction and effect of diabetes on survival. The Minnesota Heart Study. Diabetes Care 14:537-543

10. Thomas RJ, Palumbo PJ, Melton LJ et al (2003) Trends in the mortality burden associated with diabetes mellitus: a populationbased study in Rochester, Minnesota, 1970-1994. Arch Intern Med 163:445-451

11. Fang J, Alderman H (2006) Impact of the increasing burden of diabetes on acute myocardial infarction in New York City 19902000. Diabetes 55:768-773

12. Naess S, Erikson J, Midthjell K, Tambs K (2003) Diabetes mellitus and co-morbidity. Change between 1984-1986 and 1995-1997. Results of the North-Trondelag Health Study. J Diabetes Its Complicat 17:323-330

13. Rautio A, Lundberg V, Messner T, Nasic S, Stegmayr B, Eliasson M (2005) Favourable trends in the incidence and outcome of myocardial infarction in non-diabetic, but not in diabetic subjects: findings from the MONICA myocardial infarction registry in northern Sweden in 1989-2000. J Intern Med 258:369-377

14. Löwel H, Stieber J, Koenig W et al (1999) The diabetes-related risk of myocardial infarction in a region in Southern Germany. Results of the MONICA-Augsburg-studies 1985-1994. Diabetes Stoffwechs 8:11-21 (Article in German)

15. Löwel H, Lewis M, Hörmann A, Keil U (1991) Case finding, data quality aspects, and comparability of myocardial infarction registers. Results of a Southern German register study. J Clin Epidemiol 44:249-260

16. Löwel H, Meisinger C, Heier M, Hörmann A (2005) The population-based Acute Myocardial Infarction (AMI) registry of the MONICA/KORA study region of Augsburg. Gesundheitswesen 67(Suppl 1):S31-S37

17. WHO MONICA Project Principal Investigators (1988) The World Health Organisation MONICA Project (monitoring trends and determinants in cardiovascular disease): a major international collaboration. J Clin Epidemiol 41:105-114

18. Tunstall-Pedoe H, Kuulasmaa K, Amouyel P, Arveiler D, Rajakangas AM, Pajak A (1994) Myocardial infarction and coronary deaths in the World Health Organisation MONICA Project. Registration procedures, event rates, and case-fatality rates in 38 populations from 21 countries in four continents. Circulation 90:583-612

19. Löwel H, Döring A, Schneider A, Heier M, Thorand B, Meisinger C, for the MONICA/KORA Study Group (2005) The MONICA Augsburg surveys - basis for prospective cohort studies (review). Gesundheitswesen 67(Suppl 1):S13-S18

20. Meisinger C, Hörmann A, Heier M, Kuch B, Löwel H (2006) Admission blood glucose and adverse outcomes in non-diabetic patients with myocardial infarction in the reperfusion era. Int $\mathrm{J}$ Cardiol 113:229-235

21. Meisinger C, Heier M, Doering A, Thorand B, Loewel H (2004) Prevalence of known diabetes and anti-diabetic therapy between 1984/1985 and 2000/2001 in Southern Germany. Diabetes Care 27:2985-2987

22. Icks A, Moebus S, Feuersenger A, Haastert B, Jöckel KH, Giani G (2007) Diabetes prevalence and association with social statuswidening of a social gradient? German national health surveys 1990-92 and 1998. Diabetes Res Clin Pract 78:293-297

23. Le CT (2003) Introductory biostatistics. Wiley, Hoboken, San Francisco

24. Keil U (2005) The worldwide WHO MONICA Project: results and perspectives. Gesundheitswesen 67(Suppl 1):S38-S45

25. Meisinger C, Döring A, Heier M, Thorand B, Löwel H, for the MONICA/KORA Study Group (2005) Type 2 diabetes in Augsburg - an epidemiological overview. Gesundheitswesen 67 (Suppl 1):S103-S109

26. Eliasson M, Talbäck M, Rosen M (2008) Improved survival in both men and women with diabetes between 1980 and 2004-a cohort study in Sweden. Cardiovasc Diabetol 20:32

27. Rathmann W, Haastert B, Icks A et al (2003) High prevalence of undiagnosed diabetes mellitus in Southern Germany: target populations for effective screening. The KORA Survey 2000. Diabetologia 46:190-194

28. Kuch B, Heier M, von Scheidt W, Kling B, Hoermann A, Meisinger C (2008) 20-year trends in clinical characteristics, therapy, and short-term prognosis in acute myocardial infarction according to presenting electro-cardiogram: the MONICA/KORA AMI Registry (1985-2004). J Int Med 264:254-264 